\title{
Stereotactic ablative radiation therapy for spinal metastases: experience at a single Brazilian institution
}

\author{
Gustavo N. Marta ${ }^{1}$, Fernando F. de Arruda ${ }^{1}$, Fabiana A. Miranda ${ }^{1}$, Alice R.N.S. Silva ${ }^{1}$, \\ Wellington F.P. Neves-Junior ${ }^{1}$, Anselmo Mancini' ${ }^{1}$ Samir A. Hanna ${ }^{1}$, Carlos E.C.V. Abreu', João Luis F. da Silva ${ }^{1}$, \\ Jose Eduardo V. Nascimento ${ }^{1}$, Cecília Maria K. Haddad ${ }^{1}$, Fabio Y. Moraes ${ }^{2}$, Rafael Gadia ${ }^{1}$ \\ ${ }^{1}$ Department of Radiation Oncology, Hospital Sírio-Libanês, Sao Paulo, Brazil \\ ${ }^{2}$ Department of Oncology, Division of Radiation Oncology, Queen's University - Kingston Health Science Centre, Kingston, ON, Canada
}

\begin{abstract}
Background: This study aims to assess the clinical outcomes of patients with spine metastases who underwent stereotactic ablative radiation therapy (SABR) as part of their treatment. SABR has arisen as a contemporary treatment option for spinal metastasis patients with good prognoses.

Materials and methods: Between November 2010 and September 2018, Spinal SABR was performed in patients with metastatic disease in different settings: radical (SABR only), postoperative (after decompression and/or fixation surgery), and reirradiation. Local control (LC), pain control, overall survival (OS) and toxicities were reported.

Results: Eighty-five patients (corresponding to 96 treatments) with spine metastases were included. The median age was 59 years (range, 23-91). In most SABR $(82.3 \%, n=79)$ was performed as the first local spine treatment, while in 12 settings $(12.5 \%)$, fixation and/or decompression surgery was performed prior to SABR. Two-year overall survival rate was $74.1 \%$, and median survival was 19 months. The LC rate at 2 years was $72.3 \%$. With regard to pain control, among 67 patients presenting with pain before SABR, $83.3 \%$ had a complete response, $12.1 \%$ had a partial response, and $4.6 \%$ had progression. Vertebral compression fractures occurred in 10 patients (11.7\%), of which 5 cases occurred in the reirradiation setting. Radiculopathy and myelopathy were not observed. No grade III or IV toxicities were seen.

Conclusion: This is the first study presenting a Brazilian experience with spinal SABR, and the results confirm its feasibility and safety. SABR was shown to produce good local and pain control rates with low rates of adverse events.
\end{abstract}

Key words: spine metastases; treatment; radiation therapy; stereotactic body radiation therapy

Rep Pract Oncol Radiother 2021;26(5):756-763

\section{Introduction}

The spine is a usual metastatic site for several neoplasms. Extradural tumors are frequently metastatic cancer and normally develop within the vertebral bodies [1]. Metastatic spinal cord tumors can cause symptoms through invasion of normal neural components and pathways, resulting in local-regional consequences such as pain, muscular weakness, sensory dysesthesias, hypercalcemia, pathologic fracture, and spinal cord compression [2].

The objectives of treating patients with spine metastasis comprise conserving and re-establishing function, pain control, reducing the risk of

Address for correspondence: Gustavo N. Marta, Department of Radiation Oncology, Hospital Sírio-Libanês, Rua Dona Adma Jafet 91. Sao Paulo-SP 01308-050, Brazil, tel: (55 11) 33945367, e-mail: gustavonmarta@gmail.com; gustavo.marta@hsl.org.br 
skeletal-related events, and promoting local tumor control. One of the standard-of-care management options is conventional external beam radiation therapy (EBRT), which offers effective palliation of pain with low toxicity rates $[2,3]$. Nevertheless, the long-term pain and local control rates of spine metastasis patients who received conventional EBRT are low, with a 1-year local tumor relapse up to $70 \%$. Moreover, the longer the patient survival is, the higher rates of pain failure are observed $[4,5]$.

Extended life expectancy in metastatic patients is now a reality due to advances in quality of diagnostic images and oncological treatments; thus, the importance of long-term tumor and pain control has increased for many patients. In this context, stereotactic ablative radiation therapy (SABR) has arisen as a contemporary treatment option for spinal metastasis patients with good prognoses. It enables the radiation oncologist to deliver high-dose radiation (in few fractions; usually up to 5 fractions) to the specific target volume, sparing nearby organs at risk [6]. Many SABR studies have been performed and reported, however, majority come from high income countries and there is a lack of evidence of its use, safety and feasibility in low to upper middle income countries.

This study aims to assess the clinical outcomes of patients with metastatic spine cancer who underwent SABR for local treatment in a single Brazilian institution.

\section{Materials and methods}

A retrospective assessment of patients with spinal metastasis treated with SABR between November 2010 and September 2018 was carried out. Spinal SABR was performed in patients with metastatic disease in different settings: radical (SABR only), postoperative (after decompression and/or fixation surgery), and reirradiation. Based on the SINS score, if the patients had instability of the vertebral column, a surgical procedure was performed before the SABR. We considered gastrointestinal, renal, sarcoma, melanoma, and thyroid tumors as radioresistant. Demographic and clinical data of patients were obtained from medical records and treatment planning system.

This study was approved by the local institutional ethical review committee.

\section{Treatment protocol}

Stereotactic ablative radiation therapy

A full-body immobilization vac-lock was used for patients with non-cervical targets. For patients treated for cervical vertebrae, a reinforced stereotactic thermoplastic head and neck mask was employed. All patients were treated in a supine position. The clinical target volume (CTV) comprised the gross tumor and closely contiguous bony components at risk of microscopic disease infiltration, and was defined according to the Consortium Consensus Guidelines for Spinal $\operatorname{SABR}[7,8]$.

In general, magnetic resonance imaging (MRI) was used for the contouring of the tumor, spinal cord and/or cauda equina, and relevant nerve roots. For postoperative setting, CT (computed tomography) myelogram was used if the metallic artifact was severe such that the spinal cord at MRI was unrecognizable. The planning target volume (PTV) was created by adding 0 or $1 \mathrm{~mm}$ margin to the CTV. A 0 to $2 \mathrm{~mm}$ margin surrounding the spinal cord was added to define planning organ at risk volume (PRV). All patients were treated with volumetric modulated arc therapy (VMAT), RapidArc (Varian Medical Systems, Palo Alto, USA), dose delivery technique. We applied the recommendations for treatment planning and constraints for organs at risk as previously reported [6,9-11]. Online image guidance was used for each fraction using either kilovoltage cone beam CT technique (kV-CBCT), or Exactrac 6D system (BrainLAB AG, Munich, Germany).

Our institutional protocol permitted different dose schedules. This is reflection of several authors of spine SABR have used a diversity of schedules [6]; nonetheless, a clear-cut definition of the ideal number of fractions and total dose are not available. Thus, over the time, our tendency in radical and postoperative setting was to use 1 to 3 fractions, while 3 to 5 fractions were more frequently used for reirradiation setting, regardless of the tumour radioresistance features.

\section{Patient evaluation and endpoints}

The study endpoints were local control, pain control, toxicity, and overall survival.

Local progression was calculated from the first day of SABR to the date of recurrence or progression for the treated vertebral segment. Local control 
was defined as stable disease, reduction, or eradication of the tumor on images (MRI and/or CT and/or PET/CT) performed mostly every 3 to 4 months after SABR. Overall survival was calculated from the first day of SABR to the date of death from any cause. Toxicities related to SABR were evaluated according to NCI criteria (Common Terminology Criteria for Adverse Events v4.0 - CTCAE) [12]. Vertebral compression fracture (VCF) occurrences were described as the development of a new fracture or the evolution of a previous fracture in the vertebral area that received SABR based on imaging assessments.

Pain response was assessed as complete response (CR), partial response, or pain progression according to the International Consensus Guideline [13].

\section{Statistical analysis}

Continuous variables were tested for normality with the Kolmogorov-Smirnov and Shapiro-Wilk tests. Values are expressed as median and $25^{\text {th }}$ and $75^{\text {th }}$ percentiles. The categorical data is presented as absolute values and percentages and were tested using the Pearson $\chi^{2}$ test and Fisher's exact test, if applicable. The behavior of samples over time was analyzed by linear and nonlinear regression. We determined that values with $r^{2}$ values $\geq 0.8$ had good performance or linearity. The obtained equations were compared by slope values for linear regression and plateau for nonlinear regression (second-order models).

Survival analysis was performed using the Kaplan-Meier model and the estimated median values if survival was followed by the standard error of the estimate. Statistical significance was considered with $\mathrm{p} \leq 0.05$. Analyses were performed using SPSS 21.0 (IBM) and GraphPad Prism 5.0 (GraphPad).

\section{Results}

During the study period, 85 patients (corresponding to 96 treatments) with spine metastases were included. Of them, $73 \%$ had oligometastatic disease; $18 \%$, oligoprogressive and $9 \%$ were polymetastatic patients. The median age was 59 years (range, 23-91). Most SABR treatments (82.3\%, $\mathrm{n}=79$ ) were performed as the first local spine treatment (radical or de novo setting), whereas in 12 treatments (12.5\%), fixation and/or decompression surgery was performed prior to SABR (Fig. 1). Fifty
SABR treatments $(52 \%)$ were performed in patients with radioresistant tumors. All patients received systemic therapy before or after SABR. Of 8 patients treated in the re-irradiated setting, 5 received in the first treatment a dose of $5 \times 400 \mathrm{cGy} ; 3,1 \times 16$ Gy. An interval of at least one year was respected between the first and second course of radiation. Demographics and tumor characteristics, as well as prescribed doses, are presented in Tables 1 and 2.

After spinal SABR, the median follow-up period was 20 months. The overall survival rate at 2 years was $74.1 \%$ (Supplementary File - Fig. S1). The LC rate at 2 years was $72.3 \%$ (Fig. 2). With regard to pain control, of 67 patients with initial pain, $83.3 \%$ of patients had a complete response, $12.1 \%$ had a partial response, and $4.6 \%$ had progression (Fig. 3). Logistic regression for the final pain control status did not exhibit a statistically significant difference among all evaluated variables (ECOG-PS, tumor type, clinical seating and paraspinal mass, and treatment dose (Supplementary File - Table S1).

Acute or late side effects of grade $\geq 2$ were reported in $3(3.5 \%)$ of the 85 patients [grade 2 radiation dermatitis $(n=1)$, grade 2 esophagitis $(n=2)]$. VCF occurred in 10 patients (10.3\%), of which 5 cases occurred in the reirradiation setting, 2 cases in patients with lytic bone disease treated with a dose of 1 x 20 Gy and $1 \times 18$ Gy, and 3 cases in patients with baseline VCF (treated with a dose of 1 x $21 \mathrm{~Gy}, 1$ x 18 Gy and 2 x 12 Gy). Radiculopathy and myelopathy were not observed. Similarly, no grade III or IV toxicities were seen.

\section{Discussion}

To the best of our knowledge, this is the first study assessing clinical outcomes in spinal metastasis patients treated with SABR in South America. Our results emphasize that the use of SABR for spine metastasis patients is effective and safe, offering prolonged local and pain control rates with low risk of side effects. These results are comparable to European and North American centers [14].

Historically, conventional EBRT has been used as a palliative treatment for many spine metastasis patients, which has been correlated to only short-term pain management and local control rates [4]. Recently, due to advances in systemic treatments, a selection of metastatic patients live a prolonged life. 


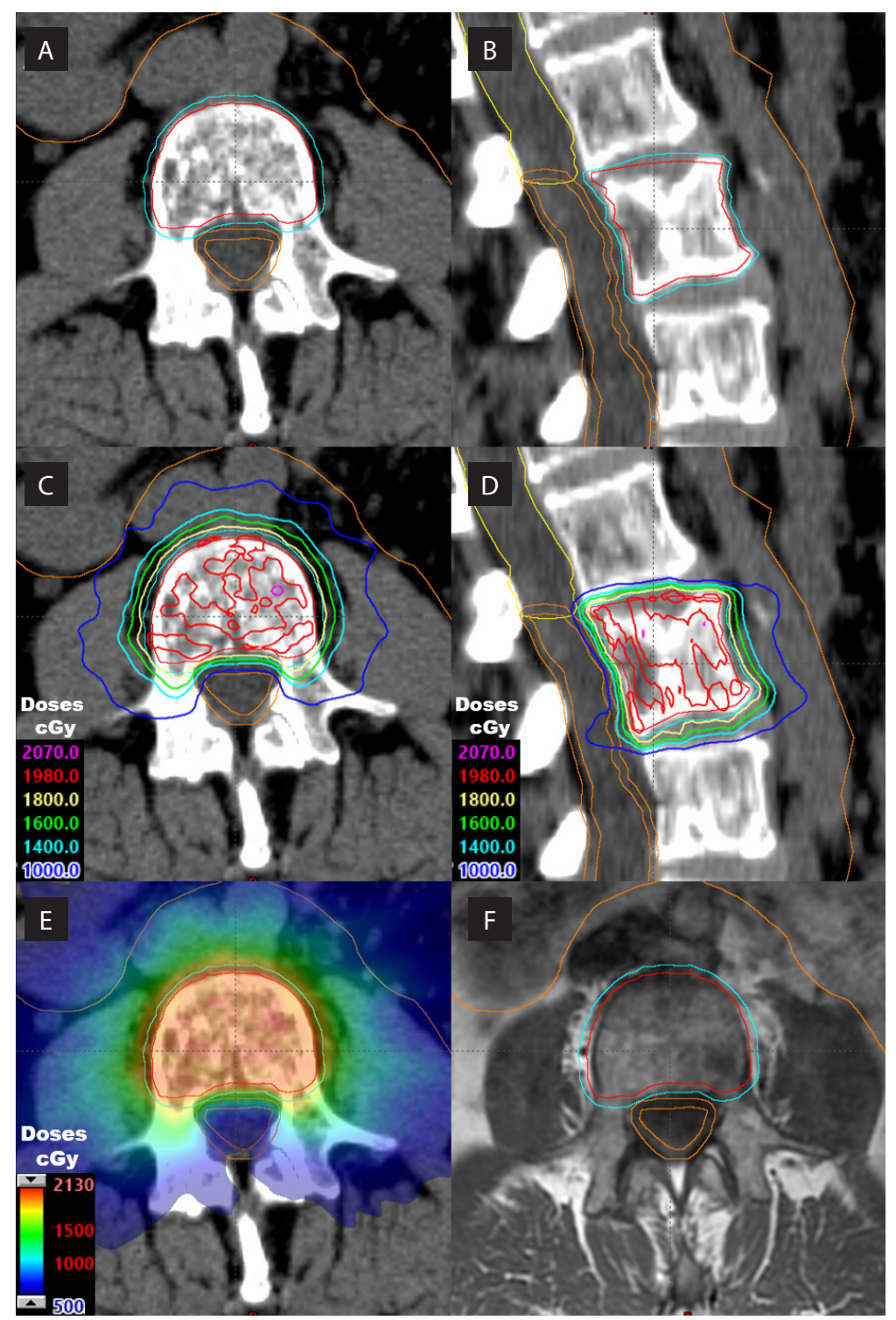

Figure 1. An example of spine SBRT plan from our institutional series. Panels A and B, the axial and sagittal views of the planning CT with CTV (red), PTV (cyan), cauda equina and its PRV (orange) and spinal canal (yellow) and its respective dose distributions at panels $C$ and $D$. Panel $E$, the color wash representation of the dose distribution on axial plane representing doses from $5 \mathrm{~Gy}$ and above. Panel F, the MR image registered to the planning CT that is required to contour the structures.

Consequently, long-term pain and local control of spinal metastasis are important treatment goals. Spinal SABR has the ability to achieve these aims, as it can deliver ablative radiation doses to the tumor while producing a steep dose gradient near organs at risk, such as the esophagus, bowels, and spinal cord, keeping doses to these areas low enough to avoid severe side effects [15].

SABR was used for oligometastases due to its impact on tumor control. Some clinical trials have indicated hopeful outcomes regarding the use of SABR for oligometastases. In fact, the idea of oligometastases is progressively recognized in modern oncology and is generating a model change in the treatment of patients with no more than 5 metastatic sites [16]. In this context, oligometastatic patients might benefit from local ablative treatments provided with radical intervention. The results of the phase II SABR-COMET trial [17] show a clinical benefit in patients with oligometastatic disease treated with SABR. The study randomized 99 patients with a controlled primary tumor and 1 to 5 metastatic lesions to palliative standard-of-care treatments alone (systemic therapy and/or conventional EBRT to symptomatic sites) or standard of care (systemic therapy) plus SABR for all metastatic 


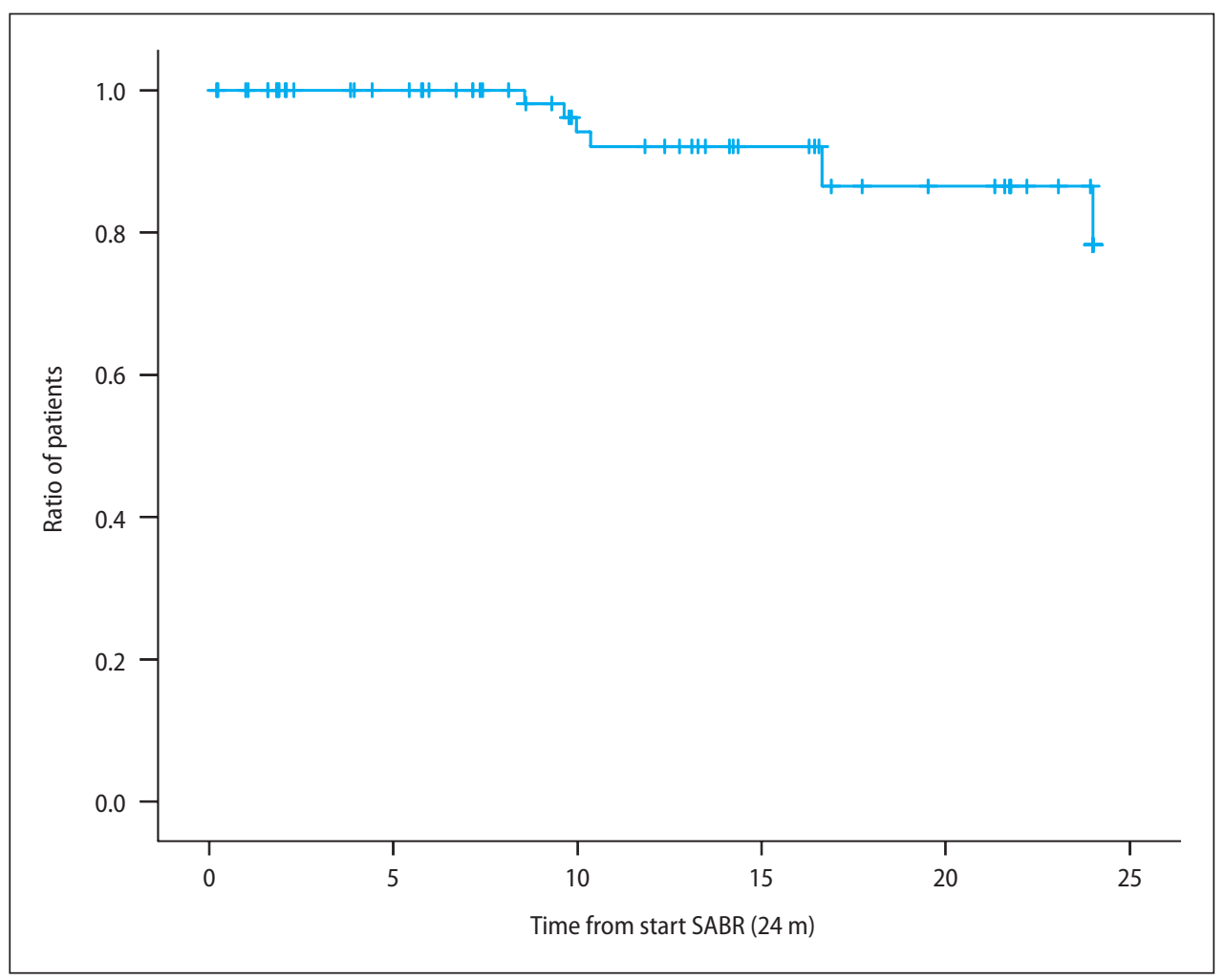

Figure 2. Local control

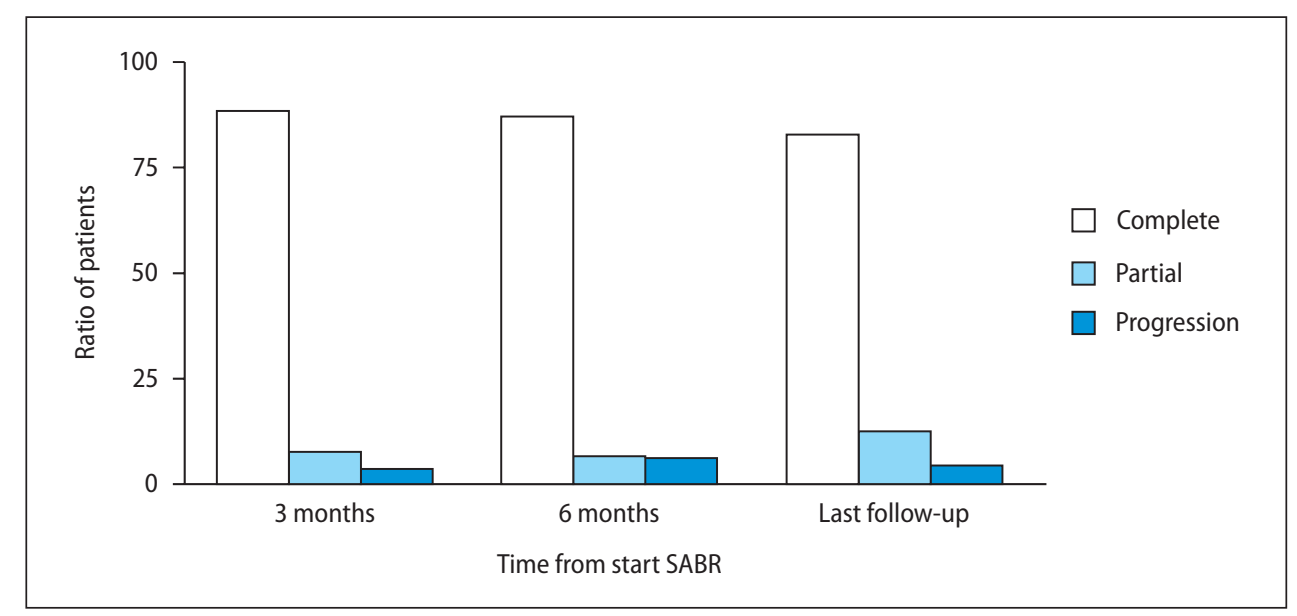

Figure 3. Pain control during the clinical follow-up among patients presenting with pain before stereotactic ablative radiation therapy $(S A B R)$

lesions, and showed a median overall survival increase from 28 to 41 months, respectively. Furthermore, there are many ongoing trials involving multiple disease-specific patients to assess the role of an ablative approach to primary and/or oligometastatic sites [18-21]. The results of all these trials are hoped to aid in clinical decisions when selecting oligometastatic patients for spinal SABR.
Notwithstanding the lack of randomized phase III trials, the efficacy and safety of SABR in spinal metastases (de novo, postoperative, and in reirradiation settings) have been mostly established in several retrospective and nonrandomized prospective studies.[14]. The first available data from a phase III Spinal SABR trial was presented by the NRG Oncology/RTOG 0631 group [22]. This trial 
compared pain relief between SABR (16 or $18 \mathrm{~Gy}$ in one fraction) and conventional EBRT (8 Gy in one fraction) for 339 spinal metastasis patients. The primary endpoint was 3-month post-treatment pain control. No difference in pain response between the groups was observed at 3 months (40.3\% for SABR vs. $57.9 \%$ for conventional EBRT; $\mathrm{p}=0.99$ ). Similarly, no significant difference between the treatment arms in rates of side effects was demonstrated. This study showed that SABR was safely executed without increasing toxicity rates, despite not showing better outcomes in the SABR group. Interestingly, our study demonstrated a higher rate of pain response at 3 months with more than $80 \%$ of patients having complete pain control. This clinical benefit remained present throughout the clinical follow-up of the patients (Fig. 3). It is important to recognize that the RTOG 0631 trial has many nuances and limitations, as it did not demonstrate suitable endpoints such as 6-, 9-, or 12-month local control or long-term pain control. Moreover, it was not expected that SABR could improve 3-month pain control over conventional EBRT in breast and prostate cancer patients who are traditionally good responders to conventional radiotherapy. When including breast and prostate cancer patients, conventional EBRT provides reasonable pain control.

CCTG SC.24/TROG 17.06 trial randomized 229 patients with painful spinal metastases to receive 20Gy in 5 fractions (conventional EBRT) or $24 \mathrm{~Gy}$ in 2 fractions (SABR). The primary endpoint was pain control (complete response) at 3 months post-radiation. Patients who underwent SABR improved the complete response rates for pain at 3 $(36 \%$ vs. $14 \% ; \mathrm{p}<0.001)$ and 6 months $(33 \%$ vs. $16 \% ; \mathrm{p}=0.004)$. There are no differences in radiation site progression-free-survival rates and side effects grade 2 or higher between the groups [23]. This is the first prospective phase III randomized trial to demonstrate that dose escalation with modern radiation therapy techniques improves pain outcomes for spinal metastases patients.

A systematic review with meta-analysis (SAFFRON study) of nonrandomized studies included 3237 patients (4911 lesions) and compared the outcomes for 1-year local control and side effects for spinal metastases patients who underwent SABR (either single or multiple-fraction) or conventional EBRT. Single dose SABR was associated with an improvement of 1-year local control compared to conventional EBRT (92.9\% vs. 81.0\%; p $=0.007$, respectively). Single dose SABR occasioned in greater local control with approximately 5\% local control advantage for every $10 \mathrm{~Gy}_{10}$ escalation [in biologically effective dose (BED) 10] compared to multiple-fraction SABR, suggesting that the SABR treatment schedule could impact the outcomes. These findings are hypothesis-generating and indicate that single dose SABR results in superior local control rate [24]. Maybe due to the limited sample size, our data did not demonstrate any association between dose and SABR for the final pain control status.

Moreover, in the SAFFRON study, higher VCF rates were noticed after single dose SABR (19.5\%) compared to multiple-fraction SABR (9.6\%; $\mathrm{p}=0.039$ ) with no association concerning VCF and dose rates. While VCF can occur after spinal SABR, when it happens, most patients are asymptomatic and medical intervention is rarely required [25]. In our study, low rates of VCF (11.7\%) were observed and this finding is comparable to the data reporting the risk for post-spinal SABR VCF that showed VCF rate of $13.9 \%$ [26]. Importantly, all patients who developed VCF in our study were at high risk for developing VCF based on known risk factors including reirradiation setting, lytic bone disease and baseline VCF [26].

Some authors have demonstrated that spine SABR succeeded in suitable local control even for radioresistant histopathology tumors $[27,28]$. The radiobiological basis for that might be justified by investigational records proposing that only after high dose of SABR, usually beyond 8 to 10 Gy per fraction, trigger pathways to increase cell death occur, mostly through the sphingomyelinase pathway [29]. In line with this rationale, our study showed good clinical outcomes in patients with radioresistant tumors.

The principal limitations of our study are associated with its retrospective design, numerous SABR dose schemes, restricted sample size, short follow-up time, and low statistical power for comparison between groups of included patients. Likewise, side effect rates should be taken with care due to the retrospective analysis, which might lead to underestimation or bias.

In a single Brazilian institution, the use of SABR in spinal metastasis patients showed high levels of long-term pain and local control with low rates of 
toxicity. These data encourage the sustained use of SABR for spinal metastatic tumors in our clinical practice and can be a motivation for other centers in developing countries.

\section{Conflict of interest}

None declared.

None declared.

\section{Funding}

\section{References}

1. Duong LM, McCarthy BJ, McLendon RE, et al. Descriptive epidemiology of malignant and nonmalignant primary spinal cord, spinal meninges, and cauda equina tumors, United States, 2004-2007. Cancer. 2012; 118(17): 4220-4227, doi: 10.1002/cncr.27390, indexed in Pubmed: 22907705.

2. Lutz S, Balboni T, Jones J, et al. Palliative radiation therapy for bone metastases: Update of an ASTRO Evidence-Based Guideline. Pract Radiat Oncol. 2017; 7(1): 4-12, doi: 10.1016/j.prro.2016.08.001, indexed in Pubmed: 27663933.

3. Silva MF, Marta GN, Lisboa FPC, et al. Hypofractionated radiotherapy for complicated bone metastases in patients with poor performance status: a phase II international trial. Tumori. 2019; 105(2): 181-187, doi: 10.5301/tj.5000658, indexed in Pubmed: 28665472.

4. Rich SE, Chow R, Raman S, et al. Update of the systematic review of palliative radiation therapy fractionation for bone metastases. Radiother Oncol. 2018; 126(3): 547-557, doi: 10.1016/j.radonc.2018.01.003, indexed in Pubmed: 29397209.

5. Klekamp J, Samii H. Surgical results for spinal metastases. Acta Neurochir (Wien). 1998; 140(9):957-967, doi:10.1007/ s007010050199, indexed in Pubmed: 9842434.

6. Osborn VW, Lee A, Yamada Y. Stereotactic Body Radiation Therapy for Spinal Malignancies. Technol Cancer Res Treat. 2018; 17: 1533033818802304, doi: $10.1177 / 1533033818802304$, indexed in Pubmed: 30343661.

7. Cox BW, Spratt DE, Lovelock M, et al. International Spine Radiosurgery Consortium consensus guidelines for target volume definition in spinal stereotactic radiosurgery. Int J Radiat Oncol Biol Phys. 2012; 83(5): e597-e605, doi: 10.1016/j.ijrobp.2012.03.009, indexed in Pubmed: 22608954.

8. Redmond KJ, Robertson S, Lo SS, et al. Consensus Contouring Guidelines for Postoperative Stereotactic Body Radiation Therapy for Metastatic Solid Tumor Malignancies to the Spine. Int J Radiat Oncol Biol Phys. 2017; 97(1): 64-74, doi: 10.1016/j.jjrobp.2016.09.014, indexed in Pubmed: 27843035.

9. Benedict SH, Yenice KM, Followill D, et al. Stereotactic body radiation therapy: the report of AAPMTask Group 101. Med Phys. 2010; 37(8): 4078-4101, doi: 10.1118/1.3438081, indexed in Pubmed: 20879569.

10. Sahgal A, Weinberg V, Ma L, et al. Probabilities of radiation myelopathy specific to stereotactic body radiation therapy to guide safe practice. Int J Radiat Oncol Biol Phys. 2013; 85(2): 341-347, doi: 10.1016/j.jirobp.2012.05.007, indexed in Pubmed: 22713832.

11. Sahgal A, Ma L, Weinberg V, et al. Reirradiation human spinal cord tolerance for stereotactic body radiotherapy. Int J Radiat Oncol Biol Phys. 2012; 82(1): 107-116, doi: 10.1016/j.ijrobp.2010.08.021, indexed in Pubmed: 20951503.

12. National Cancer Institute. Common Terminology Criteria for Adverse Events (CTCAE). http://ctep.cancer.gov/protocolDevelopment/electronic_applications/ctc.htm (22 June 2015).

13. Chow E, Hoskin P, Mitera G, et al. International Bone Metastases Consensus Working Party. Update of the international consensus on palliative radiotherapy endpoints for future clinical trials in bone metastases. Int J Radiat Oncol Biol Phys. 2012; 82(5): 1730-1737, doi: 10.1016/j. ijrobp.2011.02.008, indexed in Pubmed: 21489705.

14. Glicksman RM, Tjong MC, Neves-Junior WFP, et al. Stereotactic Ablative Radiotherapy for the Management of Spinal Metastases: A Review. JAMA Oncol. 2020; 6(4): 567-577, doi: 10.1001/jamaoncol.2019.5351, indexed in Pubmed: 31895403.

15. Vellayappan BA, Chao ST, Foote M, et al. The evolution and rise of stereotactic body radiotherapy (SBRT) for spinal metastases. Expert Rev Anticancer Ther. 2018; 18(9): 887-900, doi: 10.1080/14737140.2018.1493381, indexed in Pubmed: 29940802.

16. Guckenberger $M$, Lievens $Y$, Bouma $A B$, et al. Characterisation and classification of oligometastatic disease: a European Society for Radiotherapy and Oncology and European Organisation for Research and Treatment of Cancer consensus recommendation. Lancet Oncol. 2020; 21(1): e18-e28, doi: 10.1016/S1470-2045(19)30718-1, indexed in Pubmed: 31908301.

17. Palma DA, Olson R, Harrow S, et al. Stereotactic Ablative Radiotherapy for the Comprehensive Treatment of Oligometastatic Cancers: Long-Term Results of the SABR-COMET Phase II Randomized Trial. J Clin Oncol. 2020; 38(25): 2830-2838, doi: 10.1200/JCO.20.00818, indexed in Pubmed: 32484754.

18. Stereotacticablativeradiotherapyfor oligometastatic non-small cell lung cancer (SARON). Clinicaltrials.gov identifier: NCT02417662. https://clinicaltrials.gov/ct2/ show/ NCT02417662 (November 20, 2019).

19. Conventional care versus radioablation (stereotactic body radiotherapy) for extracranial oligometastases (CORE). Clinicaltrials.gov identifier: NCT02759783. https://clinicaltrials.gov/ct2/show/NCT02759783 (November 20, 2019).

20. Maintenance chemotherapy with or without local consolidative therapy in treating patients with stage IV non-small cell lung cancer. Clinicaltrials.gov identifier: NCT03137771. https://clinicaltrials.gov/ ct2/show/ NCT03137771 (November 20, 2019).

21. Standardofcaretherapywithorwithout stereotactic radiosurgery and/or surgery in treating patients with limited metastatic breast cancer. Clinicaltrials.gov identifier: NCT02364557. https://clinicaltrials.gov/ct2/show/ NCT02364557 (November 20, 2019).

22. Ryu S, Deshmukh S, Timmerman RD, et al. Radiosurgery Compared To External Beam Radiotherapy for Localized Spine Metastasis: Phase III Results of NRG Oncology/RTOG 
0631. Int J Radiat Oncol Biol Phys. 2019; 105(1): S2-S3, doi: 10.1016/j.jijrobp.2019.06.382.

23. Sahgal A, Myrehaug SD, Siva S, et al. CCTG SC.24/TROG 17.06: A Randomized Phase II/III Study Comparing 24Gy in 2 Stereotactic Body Radiotherapy (SBRT) Fractions Versus 20Gy in 5 Conventional Palliative Radiotherapy (CRT) Fractions for Patients with Painful Spinal Metastases. Int J Radiat Oncol Biol Phys. 2020; 108(5): 1397-1398, doi: 10.1016/j. ijrobp.2020.09.019, indexed in Pubmed: 33427654.

24. Singh R, Lehrer EJ, Dahshan B, et al. Single fraction radiosurgery, fractionated radiosurgery, and conventional radiotherapy for spinal oligometastasis (SAFFRON): A systematic review and meta-analysis. Radiother Oncol. 2020; 146: 76-89, doi: 10.1016/j.radonc.2020.01.030, indexed in Pubmed: 32114269.

25. Myrehaug S, Sahgal A, Hayashi M, et al. Reirradiation spine stereotactic body radiation therapy for spinal metastases: systematic review. J Neurosurg Spine. 2017; 27(4): 428-435, doi: 10.3171/2017.2.SPINE16976, indexed in Pubmed: 28708043.
26. Faruqi S, Tseng CL, Whyne C, et al. Vertebral Compression Fracture After Spine Stereotactic Body Radiation Therapy: A Review of the Pathophysiology and Risk Factors. Neurosurgery. 2018; 83(3): 314-322, doi: 10.1093/neuros/ nyx493, indexed in Pubmed: 29048517.

27. Bernstein MB, Chang EL, Amini B, et al. Spine Stereotactic Radiosurgery for Patients with Metastatic Thyroid Cancer: Secondary Analysis of Phase I/II Trials. Thyroid. 2016; 26(9): 1269-1275, doi: 10.1089/thy.2016.0046, indexed in Pubmed: 27334245.

28. Gerszten PC, Burton SA, Ozhasoglu C, et al. Radiosurgery for spinal metastases: clinical experience in 500 cases from a single institution. Spine (Phila Pa 1976). 2007; 32(2): 193-199, doi: 10.1097/01.brs.0000251863.76595. a2, indexed in Pubmed: 17224814.

29. Balagamwala EH, Chao ST, Suh JH. Principles of radiobiology of stereotactic radiosurgery and clinical applications in the central nervous system. Technol Cancer Res Treat. 2012; 11(1):3-13, doi: 10.7785/tcrt.2012.500229, indexed in Pubmed: 22181326. 